Original Article

\title{
Strength and muscle activities during the toe-gripping action: comparison of ankle angle in the horizontal plane between the sitting upright and standing positions
}

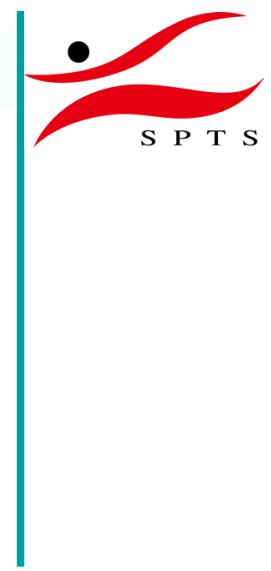

\author{
Masayuki Soma, RPT, MS ${ }^{1 *}$, Shin Murata, RPT, PhD ${ }^{2)}$, Yoshiniro Kai, RPT, PhD ${ }^{2}$, \\ Hideyuki Nakae, RPT, PhD ${ }^{1)}$, Yousuke Satou, RPT, MS ${ }^{1)}$ \\ 1) Course of Rehabilitation, Department of Health Sciences, Tohoku Fukushi University: 1-8-1 Kunimi, \\ Aoba-ku, Sendai City, Miyagi 981-8522, Japan \\ 2) Course of Physical Therapy, Department of Health Sciences, Kyoto Tachibana University, Japan
}

\begin{abstract}
Purpose] The aim of this study was to investigate whether toe grip strength and muscle activities are affected by the ankle angle in the horizontal plane in the sitting upright and standing positions. [Subjects] The subjects were 16 healthy young women. [Methods] We measured toe grip strength and the maximum voluntary contraction activities of the rectus femoris, biceps femoris, anterior tibialis, and medial head of the gastrocnemius. In addition, we calculated the percent integrated electromyography during foot gripping in 3 different ankle joint positions between the long axis of the foot and the line of progression on the horizontal plane, namely $10^{\circ}$ of internal rotation, $0^{\circ}$, and $10^{\circ}$ of external rotation. [Results] Two-way analysis of variance revealed significant differences. A significant main effect was observed in the measurement conditions for the percent integrated electromyography of the rectus femoris muscle and long head of the biceps femoris. However, two-way analysis of variance did not reveal any significant difference, and a significant main effect was not observed in toe grip strength. [Conclusion] These findings suggest that exerted toe grip strength is only slightly affected by the ankle angle in the horizontal plane in the sitting upright and standing positions. Therefore, the current measurement positions were shown to be optimal for measurement.

Key words: Toe gripping strength, Muscle activity, Measurement position
\end{abstract}

(This article was submitted Nov. 20, 2015, and was accepted Dec. 14, 2015)

\section{INTRODUCTION}

Toe gripping is defined as a complex motion that involves several muscles, similar to hand gripping. The muscles involved in the foot include the flexor pollicis brevis, flexor pollicis longus, lumbrical, flexor brevis, and flexor longus ${ }^{1}$. The position in which toe grip strength is measured has been standardized; the subject is required to sit with the trunk in the vertical position, the hip and knee joints at $90^{\circ}$, and the ankle joint in a neutral position ${ }^{1-5}$. However, there are few reports ${ }^{6-8)}$ on positions for measuring toe grip strength, and the positions used during these measurements have not been sufficiently investigated.

Souma et al. ${ }^{6}$ ) reported that the ankle angles in a neutral position and in dorsal flexion were better than plantar flexion in terms of producing the maximum toe grip strength. Nakae et al. ${ }^{7}$ reported similar levels of muscle strength exerted in the currently used measurement positions, namely sitting with the hips and knees flexed to $90^{\circ}$ and the standing position. Souma et al. studied toe grip strength under the following 3 conditions in order to determine their reproducibility: $90^{\circ}$ hip and knee flexion while sitting, $90^{\circ}$ hip flexion and knee extension while sitting, and a standing position. They reported that $90^{\circ}$ hip and

*Corresponding author. Masayuki Soma (E-mail: souma@tfu-mail.tfu.ac)

(C)2016 The Society of Physical Therapy Science. Published by IPEC Inc.

This is an open-access article distributed under the terms of the Creative Commons Attribution Non-Commercial No Derivatives (by-nc-nd) License $<$ http://creativecommons.org/licenses/by-nc-nd/4.0/>. 
knee flexion while sitting could be exerted and allowed for measurement reproducibility. However, these reports suggest that the current $90^{\circ}$ hip and knee flexion while sitting position is the most suitable limb position for measuring toe grip strength in terms of reproducibility and the ability to exhibit maximum strength ${ }^{8}$.

Ankle angle is defined as the angle formed between the long axis of the foot and the line of progression ${ }^{9}$, with hip and knee rotation and ankle abduction/adduction presumably acting as determining factors. In young adults, the normal ankle angle during walking ranges from $2.6^{\circ}$ to $17.4^{\circ 10-12)}$. The muscles involved in the exertion of toe grip strength have been shown to operate from the mid stance to the forward swing phase of the gait cycle ${ }^{13)}$, contributing to forward propulsion and stance stability, among others. Thus, the amount of toe grip strength exerted on a horizontal surface can be affected by the ankle angle. Nevertheless, Nakae et al. ${ }^{7}$ did not consider the effects of ankle angle in the horizontal plane when comparing between sitting and standing toe grip strength. Therefore, evidence on the ankle angle while sitting and standing, which focuses on the foot being on a horizontal surface, is needed.

The purposes of this study were to investigate whether toe grip strength and muscle activities are affected by the ankle angle in the horizontal plane in the sitting upright and standing positions.

\section{SUBJECTS AND METHODS}

The subjects were 16 healthy women with no known orthopedic impairments. Their age, height, and body weight (mean \pm standard deviation) were $20.8 \pm 0.7$ years, $159.3 \pm 5.8 \mathrm{~cm}$, and $50.9 \pm 6.2 \mathrm{~kg}$, respectively. The present study was approved by the Ethics Committee for Human Research of Tohoku Fukushi University (RS160102), and the subjects provided informed consent to participate.

We synchronously recorded the toe gripping strength of the dominant foot and the EMG activity of the ipsilateral lower leg to assess the muscle activities of the rectus femoris, biceps femoris, anterior tibialis, and medial head of the gastrocnemius.

Toe grip strength was measured using a toe grip dynamometer (T.K.K. 3360, Takei Scientific Instruments Co., Ltd., Niigata, Japan).

The sitting upright and standing positions were used in the measurement of toe grip strength. For the sitting upright position, the subjects were instructed to sit with the trunk vertical and the hip and knee joints at $90^{\circ}$. For the standing position, the feet were placed shoulder-width apart, and the ankle was in a neutral position with regard to dorsiflexion and plantar flexion with the arms hanging down at the sides of the trunk. To compensate for the height of the toe grip dynamometer, the height of the leg not being measured was adjusted using a supplemental platform. In each position, toe grip strength was assessed in 3 different ankle joint positions between the long axis of the foot and the line of progression in the horizontal plane, namely $10^{\circ}$ of internal rotation, $0^{\circ}$, and $10^{\circ}$ of external rotation. The sequence of the ankle positions was chosen randomly for each subject. The handle of the force meter was set on the first metatarsophalangeal joint. After a sufficient number of training trials and adequate rest, the toe gripping strength was measured twice. The maximum force was used in the analysis. In all subjects, the right toe was dominant; the dominant toe was defined as the toe used to kick a ball.

To measure the maximum voluntary contraction (MVC) activities of the tibialis anterior and medial head of the gastrocnemius muscle, each subject was instructed to sit in a chair with the ankle joint in a neutral position and to exert the maximal forces of plantar flexion and dorsiflexion in isometric contraction to resist the forces applied by the examiner in the directions of dorsiflexion and plantar flexion. To measure the MVC activity of the rectus femoris and biceps femoris muscles, each subject was instructed to sit in a chair with the hip and knee joints at $90^{\circ}$ and to exert the maximal isometric forces of knee extension and flexion in isometric contraction to resist the forces applied by the examiner in the directions of flexion and extension.

Muscular activity was measured using a surface EMG apparatus (TeleMyo G2, Noraxon USA Inc., Scottsdale, AZ, USA). After confirming adequate skin preparation (skin resistance of $<5 \mathrm{k} \Omega$ ), electrodes (Blue sensor, Ambu Inc., Ballerup, Denmark) were attached to the tibialis anterior, medial head of the gastrocnemius, rectus femoris, and biceps femoris muscles, as described by Peroto ${ }^{14}$.

The EMG signals were collected and transferred to a personal computer using analysis software (MyoResearch XP, Noraxon USA Inc., Scottsdale, AZ, USA).The bandwidth was 10-500 Hz. The EMG signal segment selected and integrated (IEMG) for analysis was the middle $1 \mathrm{~s}$ of the entire 3-s duration of continuous maximal toe grip strength exertion. The IEMG was normalized to the IEMG of the MVC of each muscle. The muscular activity used for analysis was based on the data for the maximum toe grip strength.

The SPSS software (version 12.0 for Windows, SPSS Inc., Chicago, IL, USA) was used for statistical analysis. The comparison between grip strength and muscle activities during the toe gripping action for position and measurement conditions was performed by using the two-way repeated-measures analysis of variance (ANOVA). The level of significance was set at $5 \%$.

\section{RESULTS}

Table 1 shows the average and standard deviations of the measured values in the 16 subjects. The results of the twoway ANOVA showed significant differences. A significant main effect was observed in the measurement conditions for the 
percent integrated electromyography $(\% \mathrm{IEMG})$ of the rectus femoris muscle $\left(\mathrm{F}_{(2,13)}=13.1, \mathrm{p}<0.05\right)$ and the long head of the biceps femoris $\left(\mathrm{F}_{(2,13)}=28.4, \mathrm{p}<0.05\right)$. Multiple comparisons revealed that the \%IEMG of the rectus femoris muscle was significantly higher in the standing position than in the sitting upright position and that the \%IEMG of the medial head of the gastrocnemius was significantly lower in the standing position than in the sitting upright position $(\mathrm{p}<0.05$; Table 1$)$.

The results of the two-way ANOVA did not show any significant difference. A significant main effect was not observed in toe grip strength, \%IEMG of biceps femoris, and anterior tibialis muscles.

\section{DISCUSSION}

The present study investigated whether toe grip strength and muscle activities are affected by the ankle angle in the horizontal plane in the sitting upright and standing positions. In our analysis, toe grip strength was not affected by the positions and measurement conditions. These findings suggest that exerted toe grip strength is only slightly affected by the ankle angle in the horizontal plane in the sitting upright and standing positions. Therefore, the current measurement postures were shown to be optimal for measurement.

The present study showed that toe grip strength was not affected by the positions and measurement conditions. Previous studies also demonstrated a similar level of muscle strength exerted in both measurement positions, namely sitting with the hips and knees flexed to $90^{\circ}$ and the ankle at its neutral position, and the standing position. Thus, the present study reconfirms this relationship ${ }^{7)}$. Furthermore, the present study demonstrated the new finding that toe grip strength is not affected by changes in ankle angle. Joint movement is limited by, for example, ligaments and joint capsule soft tissues, but this limitation presumably begins after muscles and other soft tissues have been extended. The subjects in this study were healthy women who demonstrated greater flexibility and range of motion in their joints than the men in previous studies ${ }^{15,16)}$. The fixed ankle angle positions of $10^{\circ}$ of internal rotation, $0^{\circ}$, and $10^{\circ}$ of external rotation may not be determining factors of toe grip strength in the present study because of the subjects' extraordinary joint flexibility, which allowed them to exert general movement through knee and hip rotation and ankle abduction/adduction. The muscles with which toe grip strength is exerted (flexor hallucis brevis, flexor hallucis longus, flexor digitorum brevis, flexor digitorum longus, and lumbricals) originate in the legs and upper feet and insert into the toes. Therefore, as hip and knee joint rotation presumably has a large effect on ankle abduction/adduction, future studies must keep hip and knee joint rotation fixed.

The present study showed that the \%IEMG of the rectus femoris muscle was significantly higher in the standing position than in the sitting upright position and that the \%IEMG of the medial head of the gastrocnemius was significantly lower in the standing position than in the sitting upright position. Previous studies also demonstrated that the \%IEMG of the medial head of the gastrocnemius was significantly lower in the standing position than in the sitting upright position ${ }^{7)}$. Thus, the present study reconfirms this correlation. In addition, the present study revealed was as new knowledge that the \%IEMG of the rectus femoris muscle was significantly higher in the standing position than in the sitting upright position. The activities of the quadriceps muscle increase more easily in the standing position than in the sitting upright position because this muscle is an antigravity muscle, the activities of which are controlled in the standing position.

In addition, we inferred the following observations: When measuring toe grip strength in the standing position, the center of gravity can shift posteriorly as toe grip strength is exerted, requiring the subject to bend the forefoot (including the toes). Both the ankle and hip joints are used to compensate for this posterior center of gravity, which results in knee joint extension. The joints should be stabilized when exerting muscular force; therefore, we feel that by fixing the knee joint in the direction of extension, great toe gripping power can be exerted. Thus, it appears that the quadriceps activity increases to fix the knee in an extended position, that is, to stabilize the knee.

The present results show that the current measurement positions, namely sitting with the trunk vertical and the hip and knee joints at $90^{\circ}$ angles and the ankle joint at neutral position, was optimal for measurement because it has the added advantage of facilitating measurement in subjects who are unable to maintain a stable standing posture or have difficulty in

Table 1. Comparison of each positions and each measurement conditions

\begin{tabular}{lrrrrrrr}
\hline & \multicolumn{3}{c}{ Sitting upright position } & \multicolumn{2}{c}{ Standing position } & Main effect \\
\cline { 2 - 7 } & $\begin{array}{l}\text { Internal } \\
\text { rotation }\end{array}$ & $\begin{array}{r}\text { Neutral } \\
\text { position }\end{array}$ & $\begin{array}{r}\text { External } \\
\text { rotation }\end{array}$ & $\begin{array}{r}\text { Internal } \\
\text { rotation }\end{array}$ & $\begin{array}{c}\text { Neutral } \\
\text { position }\end{array}$ & $\begin{array}{c}\text { External } \\
\text { rotation }\end{array}$ & $\begin{array}{c}\text { Position } \\
\text { Measurement } \\
\text { conditions }\end{array}$ \\
\hline Toe gripping strength (kg) & $20.0 \pm 3.3$ & $20.2 \pm 2.6$ & $21.1 \pm 3.7$ & $20.2 \pm 3.6$ & $20.5 \pm 3.2$ & $20.3 \pm 3.5$ & \\
Rectus femoris muscle (\%IEMG) & $3.1 \pm 2.0$ & $3.1 \pm 2.0$ & $3.0 \pm 1.6$ & $10.3 \pm 8.0$ & $11.3 \pm 9.4$ & $10.7 \pm 8.6$ & $* *$ \\
Long head of the biceps femoris & $21.6 \pm 12.8$ & $23.1 \pm 16.1$ & $23.0 \pm 17.1$ & $15.5 \pm 8.0$ & $16.9 \pm 7.2$ & $17.0 \pm 9.8$ & \\
muscle (\%IEMG) & $23.1 \pm 16.0$ & $23.1 \pm 16.1$ & $23.9 \pm 16.4$ & $27.2 \pm 18.0$ & $32.9 \pm 24.7$ & $30.0 \pm 19.7$ & \multirow{2}{*}{ Tibialis anterior muscle (\%IEMG) } \\
$\begin{array}{l}\text { Medial head of the gastrocnemius } \\
\text { muscle (\%IEMG) }\end{array}$ & $54.3 \pm 23.9$ & $59.7 \pm 20.6$ & $61.4 \pm 23.1$ & $31.7 \pm 11.3$ & $33.8 \pm 10.4$ & $33.9 \pm 10.7$ & $* *$ \\
\hline
\end{tabular}

\footnotetext{
Mean \pm standard deviation (SD). ${ }^{*} \mathrm{p}<0.05$
} 
standing.Our study has some limitations. First, we were unable to avoid various common problems that negatively affect surface EMG, such as resistance of the skin, artifacts, and the effects of proximal muscles. Second, only healthy young women participated; thus, it is difficult to extrapolate our findings to the general population. Future studies should include healthy young men and other age groups.

\section{REFERENCES}

1) Murata S, Kutuna T: Prevention of fall in the elderly disabled at home-Toe grip strength exercise- - J Jpn Acad Home Care, 2004, 7: 67-74.

2) Uritani D, Fukumoto T, Matsumoto D: Intrarater and Interrater reliabilities for toe grip dynamometor. J Phys Ther Sci, 2012, 24: 639-643. [CrossRef]

3) Kito N, Ihara H, Miwa M, et al.: Effects of toe motion exercise to prevent falls in the elderly. Phys Ther Jpn, 2001, 28: 313-319.

4) Fukuda I, Kobayashi R: The effects of toe-grip training in healthy young adults. Phys Ther Jpn, 2008, 35: 261-266.

5) Soma M, Murata S, Kai Y, et al.: Activity of the femoral muscles during toe-gripping action. J Phys Ther Sci, 2014, 26: 1619-1621. [Medline] [CrossRef]

6) Soma M, Murata S, Kai Y, et al.: Effect of joint position on foot-grip strength. Japanese Journal of Health Promotion and Physical Therapy, 2013, 3: 21-23. [CrossRef]

7) Nakae H, Murata S, Kai Y, et al.: Comparison of the lower limbs muscular muscular activity of toe grip and the toegripping strength in sitting upright position and the standing position. Japanese Journal of Health Promotion and Physical Therapy, 2013, 3: 11-14. [CrossRef]

8) Soma M, Murata S, Kai Y, et al.: An examination of limb position for measuring toe-grip strength. J Phys Ther Sci, 2014, 26: 1955-1957. [Medline] [CrossRef]

9) Murray MP, Kory RC, Clarkson BH, et al.: Comparison of free and fast speed walking patterns of normal men. Am J Phys Med, 1966, 45: 8-23. [Medline] [CrossRef]

10) Rigas C: Spatial parameters of gait related to the position of the foot on the ground. Prosthet Orthot Int, 1984, 8: 130-134. [Medline]

11) Murray MP, Kory RC, Sepic SB: Walking patterns of normal women. Arch Phys Med Rehabil, 1970, 51: 637-650. [Medline]

12) Murata S, Kutsuna T, Kitayama T: Difference in optimal walk and fastest walk: analysis by GAITRite. Rigakuryoho kagaku, 2004, 19: 217-222. [CrossRef]

13) Perry J: Gait analysis normal and pathological function. Tokyo: Ishiyaku Shuppan, 2009, pp 43, 96.

14) Aldo O: Perotto (translation: Hakumori R): Anatomical guide for electromyographer-The limbs and trunk—3rd ed. Tokyo: Nishimura Company, 2007, pp 140-155.

15) Grimston SK, Nigg BM, Hanley DA, et al.: Differences in ankle joint complex range of motion as a function of age. Foot Ankle, 1993, 14: 215-222. [Medline] [CrossRef]

16) Riemann BL, DeMont RG, Ryu K, et al.: The effects of sex, joint angle, and the gastrocremius muscle on passive ankle joint complex stiffness. J Athl Train, 2001, 36: 369-375. [Medline] 